\title{
El derecho de acceso a la educación de alumnos inclusivos en tiempos de COVID-19
}

\author{
The right of access to education for inclusive students in times of COVID-19
}

O direito de acesso à educação para alunos inclusivos nos tempos de COVID-19

Aracelli del Carmen Gonzales-Sánchez

dgonzalessa@ucvvirtual.edu.pe

https://orcid.org/0000-0003-0028-9177

Universidad César Vallejo- Ate, Perú

y CIFE-Cuernavaca, México

\author{
Gustavo Zenobio Apolinario Olivera \\ g.apolinario2021@gmail.com \\ https://orcid.org/0000-0001-5731-6469
}

Universidad César Vallejo - Los Olivos, Perú

Richard Cervantes Juro

perurichard1@gmail.com

https://orcid.org/0000-0003-4486-3353

Universidad César Vallejo - Los Olivos, Perú

\section{RESUMEN}

El acceso a la educación es un derecho, además una obligación para los gobiernos en garantizar un servicio educativo de calidad que incluya a todos y todas los estudiantes, sea la condición que tengan, una sociedad democrática debe ser equitativa e igualitaria; sin embargo la realidad ha demostrado el difícil proceso para los estudiantes inclusivos de insertarse en el sistema educativo, con iguales condiciones, sin embargo, esta situación se complicó con la emergencia sanitaria producto del COVID-19, develó múltiples dificultades y demostró la complicada situación de estos alumnos, el objetivo de estudio fue explorar cual es la información que brinda la literatura académica con respecto al acceso a la educación en alumnos inclusivos, se empleó como metodología el enfoque cualitativo de revisión bibliográfica, se pudo concluir que fue difícil brindarles aprendizaje de calidad, en un inicio se sintieron excluidos y gradualmente se dieron cambios para mejor su atención.

Palabras clave: Aprendizaje; Derecho; Educación; Inclusión, Pandemia

ABSTRACT

RESUMO

Access to education is a right, as well as an obligation for governments to guarantee a quality educational service that includes all and all students, whatever their condition, a democratic society must be equitable and egalitarian; However, reality has shown the difficult process for inclusive students to enter the educational system, with the same conditions, however, this situation was complicated by the health emergency caused by COVID-19, revealed multiple difficulties and demonstrated the complicated situation of These students, the objective of the study was to explore what information the academic literature provides regarding access to education in inclusive students, the qualitative approach of bibliographic review was used as a methodology, it was possible to conclude that it was difficult to provide them with quality learning At first, they felt excluded and gradually changes were made to improve their care.

Key words: Learning; right; Education; Inclusion, Pandemic
O acesso à educação é um direito, assim como uma obrigação dos governos de garantir um serviço educacional de qualidade que inclua todos e todos os alunos, qualquer que seja sua condição, uma sociedade democrática deve ser eqüitativa e igualitária; Porém, a realidade tem mostrado o difícil processo de entrada dos alunos inclusivos no sistema educacional, com as mesmas condições, porém, esta situação foi complicada pela emergência sanitária provocada pelo COVID-19, revelou múltiplas dificuldades e evidenciou a situação complicada destes alunos, o objetivo do estudo foi explorar quais as informações que a literatura acadêmica fornece sobre o acesso à educação de alunos inclusivos, a abordagem qualitativa da revisão bibliográfica foi utilizada como metodologia, foi possível concluir que foi difícil proporcionar-lhes uma aprendizagem de qualidade. primeiro eles se sentiram excluídos e, gradativamente, foram feitas mudanças para melhorar seu atendimento.

Palavras-chave: Aprendendo; Certo; Educação; Inclusão, Pandemia 


\section{INTRODUCCIÓN}

Una sociedad democrática e inclusiva, es aquella que convive con todos ciudadanos en igualdad de condiciones, lamentablemente se han dado prácticas de exclusión cuando no se asemeja en apariencia a lo socialmente aceptable, en tal sentido históricamente el haber nacido con una limitación cognitiva, sensorial o física, convirtió a la persona en anormal, dándose situaciones de rechazo, porque se defendió la perfección; en tal sentido con el correr de los siglos se asumieron diversas posturas de cómo concebir al otro cuando no es normal.

Para Nunes et al. (2015) la mayor dificultad que ha representado el trato a las personas con discapacidad, se debió a la influencia de factores culturales y religiosos, la imagen que se les tuvo fue de tontos, maldecidos, o producto de una acción que iba contra los designios divinos (Balanta et al., 2014) el surgimiento de un discurso científico, genero una forma más adecuada de comprender la discapacidad, desde un punto fisiológico, esto contribuyó a restar importancia a los prejuicios religiosos.

Es en este sentido que el siglo XX, significó una nueva forma de comprender y entender a las personas con discapacidad, Hernández (2015) sostuvo tres modelos: el médico, en el cual el discapacitado es un enfermo, podrá ser útil a la sociedad cuando sea sujeto de rehabilitación; el segundo sería el modelo social, en el cual depende de las relaciones sociales y la aceptación; finalmente el tercer modelo fue el de "biopsicosocial- que integra los modelos médico y social - ha pasado de la discapacidad como deficiencia y responsabilidad individual a la discapacidad como diferencia y reconocimiento social" (Hernández, 2015, p. 50).

\section{La discapacidad como derecho social}

Diniz et al. (2009) señalaron que, Inglaterra a partir de los años 1970, se dieron los primeros reconocimientos de la dicacidad como derecho social, así mismo en esta década se iniciaron los primeros reclamos de los derechos sociales, de tal manera que surgieron activistas como "Alan Reich, Kathryn McGee, Ed Roberts, John Tyler, Diana Braun, Kathy Conour y Gabriela Brimmer" (Valencia, 2018, p. 286); que reclamaron, por igualdad de trato, además que la sociedad estuviera preparada para permitirles de ejercer su ciudadanía sin limitaciones. Es en esta línea que la Organización de Naciones Unidas (ONU) el año 1982 aprobó el Programa de Acción Mundial para las personas con discapacidad, motivando a los países miembros promover la igualdad de condiciones, se establece como fecha emblemática el día 36 de diciembre en donde se recuerda nivel internacional a las personas con discapacidad, mientras que en Perú esta fecha serás objeto de concientización el 16 de octubre.

\section{Escuela inclusiva en Perú}

En el transcurso de esta investigación se ha accedido a limitada información de cómo ha evolucionado históricamente la atención a las personas con discapacidad, sin embargo, el gran logro que se consideró como parte de este estudio es el brindar una visión panorámica del ingreso normativo en la escuela. Es importante precisar que el termino más adecuado es de estudiantes con necesidades especiales, en los que se ubican aquellos con ligeros temas cognitivos, sensoriales, físicos y de alto rendimiento, en tal sentido se acuña un importante termino que es a la escuela inclusiva, como aquella que también ofrece una educación de calidad, atendiendo a la diversidad 
de los estudiantes, mediante un proceso de adaptación (Echeita y Ainscow,2011) en tal sentido esto involucra a los profesionales que brindan las experiencias de aprendizaje en las, los cuales deben adaptarlos de acuerdo a los alumnos: para Aguinaga et al. (2018) las actividades de inclusión educativa constituyó un desafío para quienes son profesionales de la educación. De acuerdo a Llancavil (2016) una escuela inclusiva es aquella donde la enseñanza se adapta a los alumnos, se les permite vivir en comunidad; ofreciéndoles una educación de calidad.

En los últimos años en Perú, la forma de cómo se imparte la educación en la escuela ha cambiado, a los docentes les costó comprender las nuevas orientaciones de como impartir el servicio educativo, consistió en abandonar la enseñanza tradicional y cognitiva. La nueva escuela buscó valorar las capacidades y competencias en los estudiantes, promoviendo en las aulas el trabajo cooperativo, el pensamiento crítico, exponiéndolos a situaciones retadoras de las cuales ellos deberán brindar soluciones.

El impulsar una educación inclusiva en nuestro país tiene como antecedentes las recomendaciones que hizo la Organización de las Naciones Unidas para la Educación, la Ciencia y la Cultura (UNESCO) desde 1990 ha promovido, primero fue el enunciado de una Educación Para Todos (EPT), años más tarde en la misma línea para reafirmar la inclusión, se dieron acuerdos internacionales, como la Declaración de Salamanca: Conferencia Mundial sobre Necesidades Educativas Especiales: Acceso y Calidad en el año 1994 y la Conferencia que se tituló "La educación inclusiva: El camino hacia el futuro" en el 2008.
Actualmente está vigente la Declaración de Incheon (2016) realizada en la República de Corea, este documento suscrito por 160 países el año 2015, brinda las pautas de la educación que se quiere para el año 2030, esta debe ser inclusiva, equitativa y de calidad. Además, fomenta el aprendizaje a lo largo de la vida para todos.

El Perú como miembro de la UNESCO, debió realizar los ajustes necesarios para que la educación inclusiva sea de interés nacional y una política pública. El año 2003 el Ministerio de Educación promulgó el D.S. 026-2003-ED que declaró la "Década de la Educación Inclusiva 2003-2012", para fortalecer políticas en favor de la educación inclusiva, razón por la cual las aulas de Educación Básica Regular, debían darse a la apertura a los alumnos con necesidades especiales, en este grupo consideramos a los estudiantes que tienen discapacidad intelectual leve y moderada, motora y sensorial; también se incluye a los niños con talento o superdotación.

En la Ley 27050 publicada en el diario Oficial el Peruano, el 24 de setiembre del 2012, en el artículo 35 se señala el derecho a la educación de las personas con discapacidad, siendo el Ministerio de Educación (Minedu) el responsable de regularla y garantizarla, en el artículo 36 la accesibilidad al sistema educativo y en el artículo 37 la calidad del servicio educativo. Es en esta línea que, el Currículo Nacional de Educación Básica (CNB) (Minedu, 2016), incluye el enfoque transversal Inclusivo o de Atención a la diversidad que debe ser incorporado a todas las áreas. Así mismo el Proyecto Educativo Nacional (PEN), (CNE, 2019) puesto en valor hasta el 2036, otorga a la educación inclusivo un valor normativo, priorizado por el Estado peruano. 
De acuerdo a lo líneas atrás mencionado surgieron como interrogantes en esta investigación ¿de qué manera se imparte la educación a los alumnos con NEE durante el CPVID-19? ¿cuáles serán las acciones que estarán implementando los países con respecto a los alumnos inclusivos? ¿cómo se sentirán los padres pata poder ayudar a sus hijos en este contexto de la emergencia sanitaria? Por lo que se formuló como propósito de estudio explorar cual es la información que brinda la literatura académica con respecto al acceso a la educación en alumnos con NEE en el contexto de la emergencia sanitaria por el COVID-19. Este artículo se considera importante porque brinda de manera sistematizada a los investigadores información temprana sobre el acceso a la educación en alumnos de NEE durante la pandemia.

\section{MÉTODO}

La metodología empleada en este estudio fue de enfoque cualitativo bajo una revisión bibliográfica, en la cual se recopiló, analizó, sintetizó y discutió, la información publicada sobre el acceso a la educación en alumnos de NEE durante la pandemia.; por tanto, se consultó las bases de datos: SCOPUS, Web of Science (WOS). ERIC Y SCIELO, tomando como referencia 12 artículos científicos. Además, se recurrió a documentos normativos peruanos con respecto a la educación inclusiva emitidos por el Minedu y la Defensoría del Pueblo, Se consideró como criterio de inclusión que los artículos sean del año 2020 y 2021; e incluyeran en su contenido dos aspectos relevantes: educación inclusiva $\mathrm{y}$ la pandemia. Además, se tuvo como criterio de exclusión que solo se abordara el aspecto educativo desde la EBR, se desestimó los aportes académicos desde la perspectiva de Escuelas Técnico Productivas y universidades. La literatura consultada permitió establecer tres tópicos en los cuales coincidieron los autores; el apoyo de los padres, las dificultades de los docentes y la implementación de política públicas para estudiantes inclusivos. Además, para sistematizar la información se construyeron fichas de contenidos, de cada uno de los 12 artículos consultados.

\section{DESARROLLO Y DISCUSIÓN}

La literatura académica revisada en los artículos científicos permitieron explorar cómo se ha abordado el tema del acceso del derecho a la educación a los estudiantes inclusivos en distintos países, que permitió establecer similitudes, de tal manera se encontraron iguales dificultades, sea en América, Europa o África.

En primer lugar, las dificultades que afrontaron los padres en brinda a poyo a sus hijos no solo se presentó en Perú; también fue un problema en otros países. La implementación de la educación a distancia o remota, durante la pandemia del COVID-19, significó que los hogares se convirtieran en escuelas, esto demando mayor exigencia en los padres los cuales, tuvieron que afrontar además del confinamiento obligatorio o cuarentena, las dificultades económica que generó la pandemia e incertidumbre por esta enfermedad, fue complicado la regulación de las emociones, Abdelfattah et al. (2021) estudiaron este problema en países árabes, evidenciando la sensación de frustración en los padres de familia al no poder ayudar a sus hijos inclusivos, Pozas et al. (2021) realizaron un estudio comparativo entre México y Alemania, dos países con sistemas educativos distintos; sin embargo durante la pandemia del COVID-19, los padres coincidieron en las limitaciones pedagógicas y de 
adaptación al nuevos sistema educativo para ayudar s sus hijos con NEE. Asbury et al., (2021) sostuvieron que, los padres de estos estudiantes se sienten más vulnerables y propensos a tener afecciones en su salud mental. Por otro lado, Tabatadze y Chachkhiani (2021) quienes estudiaron este tema en Georgia, determinaron que, la pandemia evidenció la importancia del apoyo de los padres como aliados, permitiendo mejor los aprendizajes; sin embargo, existen aspectos negativos, siendo necesario poner límites; asimismo, brindar las pautas claras de su colaboración.

El segundo aspecto en el cual se encontraron coincidencias en la literatura revisada fue el tema del apoyo de los docentes a estudiantes inclusivos, ellos afrontaron en Perú severas dificultades porque la educación remota o a distancia, significó una nueva experiencia que permitió continuar con el servicio educativo. En Perú se emitieron normativas para instituciones degestión pública y privada, comola RV Nº 093 (Minedu, 2020) “Orientaciones pedagógicas para el servicio educativo de Educación Básica durante el año 2020 en el marco de la emergencia sanitaria por el Coronavirus COVID-19", las cuales brindaron los lineamientos a todos los actores de la comunidad educativa de cómo se desarrolló la educación durante la emergencia sanitaria, esta situación develó una realidad que ya existió cuando la educación era presencial; sin embargo fue poco atendida por las autoridades del Minedu, se manifestó la poco preparación en los docentes para poder atender a estudiantes inclusivos y brindarles una educación de calidad.

Esta situación se debe porque a partir del 2003 de dieron políticas pública en Perú para implementar la educación inclusiva en aula de EBR, con "Década de la Educación Inclusiva 2003-2012"; por esta razón las universidades debieron adecuar sus mallas curriculares de las carreras universitarias de educación incluyendo cursos para atención a la diversidad, en tal sentido existe un importante números de docentes egresado hasta antes del 2012, que no cuentan con esta preparación, debieron por su cuenta llevar cursos sobre inclusión que fortalezcan su práctica pedagógica Calvo (2013) sostuvo que una preocupación de la formación docente en América Latina, fue que existen personas las cuales ejercen esta labor sin tener formación pedagógica, la preparación debe ser una política de Estado continua y permanente para atender a los alumnos inclusivos. Herrera et al. (2018) estudiaron esta problemática en Ecuador coincidieron en la necedad de mejorar las mallas curriculares universitarias, además determinaron que la formación profesional de los docentes en educación se da de manera general u obteniendo especializaciones.

Gallego-Ortega y Rodríguez-Fuentes (2021) señalaron la importancia en la actitud de los docentes al atender a este grupo de alumnos, se debe evitar colocar estigmas a los estudiantes, tiene que sentirse motivado y no obligados en cumplir estas labores. De forma contrario Sevilla et. al, (2018) encontraron en su investigación de manera general una predisposición negativa de los docentes en atender a los alumnos inclusivos, entre los factores que generan el rechazo se encontraron la atención a los padres y la preparación de material académico adecuado, esto se debe a la falta de preparación académica. Rojo-Ramos et al. (2021) exploraron este tema en España, señalaron que la formación de los docentes al especializar en atención a la diversidad, contribuyó a brindar una educación de calidad, además las capacitaciones deben ser permanentes. 
Además, otro problema que afrontaron los docentes fue la falta de preparación en competencias digitales, necesaria en el nuevo sistema educativo, en Perú se colocó a disposición de los maestros la plataforma PerúEduca, con cursos y recursos para mejorar su práctica profesional en el nuevo contexto.

El tercer aspecto que destacó la consulta de la literatura académica fue las políticas que implementaron los gobiernos, para implementar el servicio educativa en estudiantes inclusivos. En Perú como parte de las medidas que se dieron por la pandemia se promulgó (Peruano, 2020) el DL N ${ }^{\circ} 1468$ "Disposiciones de prevención y protección para las personas con discapacidad ante la emergencia sanitaria ocasionada por el COVID-19", en tal sentido se debía garantizar la continuidad de extruidos en aquellos estudiantes inclusivos, se brindaron las recomendaciones a los docentes para atender a estudiantes con esta condición mediante adaptaciones, buscar el apoyo de la familia y mantenerlos informados del progreso de sus hijos. Sin embargo, la realidad comprobó que poco se pudo hacer para la atención de estos alumnos, como lo demostró Defensoría del Pueblo (2020) la pandemia evidenció dificultades que ya tenía la atención a los estudiantes con NEE, como era la falta de personal preparado el cual ayudara no solo a los docentes, sino también a los padres; así como la implementación del programa "Aprendo en casa", al principio no los involucró, gradualmente se realizaron adecuaciones.

Semejantes dificultades se dieron en otros países, Kamga (2020) investigó esta problemática en Sudáfrica, concluyendo que en la práctica los estudiantes en esta condición fueron excluidos, a pesar que el gobierno mostró predisposición de dar recursos tecnológicos para poder acceder a la educación remota o a distancia, no ayudó mucho porque los equipos no están adaptados para ser utilizados por estudiantes en esta condición. Murillo y Duk (2020) describieron en Chile al igual que varios países de América Latina, las dificultades del acceso a la educación durante esta coyuntura también se deben a las brechas sociales, de no contar los hogares con disponibilidad de internet e incluso de servicio eléctrico, hacer imposible puedan emplear los recursos tecnológicos o seguir la emisión de los programas "Aprendo en casa". Moreno Rodríguez et al. (2020) de acuerdo al estudio que realizaron en España, determinaron que los padres necesitaban sentirse acompañados en este proceso, el reto de trasladar el aula a sus hogares. La necesidad de sentirse escuchados, contar con canales de comunicación mediante cualquier medio de conectividad, similar situación sucedió en Perú que no fue exclusivo de los alumnos con NEE; se presentaron múltiples conflictos codo dudar su este sistema educativo era de calidad podrían los estudiantes aprender, bajar los costos de colegios privados porque la educación implicaba menos gastos al no emplear los locales, esta situación conflictiva exigió el liderazgo de los directores desde la gestión (Gonzales-Sánchez et al., 2021).

Por otro lado, Jia y Santi (2021) quienes estudiaron el tema en China, describieron que las políticas públicas implementadas fueron inadecuadas, se alejaron del principio en considera la educación como derecho en estudiantes inclusivos, de tal manera en el futuro las autoridades deben ser más cuidadosas al emitir normativas que incluyan a todos y todas, esto fue una importante lección que dejó la pandemia. En esta línea el informe (CEPAL-UNESCO 2020) históricamente este grupo 
es excluido, los Estados deber realizar cambios significativos para mejorar esta situación, en el contexto de la emergencia sanitaria recomendaron que los país de América Latina y el Caribe devoran priorizar la atención de estos estudiantes para evitar que se desvinculen con la escuela, estableciendo medidas pedagógica y flexibles en los escolares de esta condición que les permita continuar con el servicio educativo.

\section{CONCLUSIÓN}

Teniendo en consideración que, el objetivo de estudio fue explorar sobre la información que brindó la literatura académica con respecto al acceso a la educación en alumnos inclusivos en Perú y otros países durante el contexto de la emergencia sanitaria por el COVID-19, permitió concluir que a partir de fines del 2020 aparecieron publicaciones las cuales asordaron este tema; la información académica aun es limitada, se infirió que la comunidad académica conforme pase más tiempos y estudie los efectos emitirá más información.

De acuerdo a los a los artículos consultados, se concluyó también que existieron tres coincidencias las cuales preocuparon a los investigadores: las limitaciones que afrontaron los padres de familia en brindar apoyo a los alumnos inclusivos durante el sistema de educación remota o a distancia, las dificultades de los docentes de no poder atender adecuadamente a sus alumnos con esta condición, debido a la carencia de competencias digitales y preparación pedagógica en la atención a la diversidad y el tercer aspecto fue la poca predisposición de los gobiernos en emitir políticas públicas más adecuadas que incluyeran a estos alumnos. En tal sentido, fue difícil brindarles aprendizaje de calidad a los estudiantes inclusivo, en un inicio se sintieron excluidos y gradualmente se dieron cambios para mejor su atención. Los distintos gobiernos intentaron dotar de recursos tecnológicos a los padres de estudiantes inclusivos, para que continúen su formación académica, sin embargo, la carencia de competencias digitales, tanto de los padres y docentes, obstaculizaron la correcta implementación de la educación a distancia o remota; esta realidad los obligó a tomar medidas correctivas para mejorar el acceso a la educación en los alumnos inclusivos.

El año 2021 demostró mejoras en la atención a la diversidad, como es la implementación de traductores en señas para los distintos programas "Aprendo en casa", impulsado por los países; sin embargo, faltan estrategia para la atención de estudiantes sordos, o limitaciones cognitivas. No hay duda que el acceso a la educción es un derecho una misión la cual deben implementar los gobiernos como políticas públicas, adecuaciones y recursos para que todas y todos sin distinción reciban una educación de calidad.

\section{REFERENCIAS}

Abdelfattah, F., Rababah, A., Alqaryouti, I., Alsartawi, Z., Khlaifat, D., y Awamleh, A. (2021). Exploring feelings of worry and sources of stress during covid-19 pandemic among parents of children with disability: A sample from arab countries. Education Sciences, 11(5). https://doi. org/10.3390/educsci11050216

Aguinaga, S., Velásquez, M., y Rimari, M. (2018). Modelo contextualizado de inclusión educativa. Revista Educación, 42(2), 2215-2644

Asbury, K., Fox, L., Deniz, E., Code, A., y Toseeb, U. (2021). How is COVID-19 Affecting the Mental Health of Children with Special Educational Needs and Disabilities and Their Families? Journal of Autism and Developmental Disorders, 51(5), 1772-1780. https://doi.org/10.1007/ s10803-020-04577-2 
Balanta Cobo, P., Estrada Hernández, R., Rodríguez Díaz, F., y Rodríguez Jiménez, N. (2014). Evolución del concepto de discapacidad: estado del arte de las investigaciones sobre el tema desde una perspectiva interdisciplinar. In Configuración de la noción de discapacidad en un contexto de vulnerabilidad en Bogotá. Análisis interdisciplinar. https://doi. org/10.7476/9789587384758.0006

Calvo, G. (2013). La Formación De Docentes Para La Inclusión Educativa. 6(1), 19-35. https://doi. org/10.22235/pe.v6i1.525

CEPAL-UNESCO. (2020). La educacion en tiempos de la pandemia COVID-19. Comisión Económica Para América Latina y El Caribe, Santiago Oficina Regional de Educación Para América Latina y El Caribe de La Organización de Las Naciones Unidas Para La Educación La Ciencia y La Cultura, 11, 11-13. https:// unesdoc.unesco.org/ark:/48223/pf000037407 5 ? posInSet $=1 \&$ queryId $=9$ ccf $4 a 39-7 \mathrm{c} 50-43 \mathrm{e} 4-$ 856b-a09632daa7a2

CNE. (2019). Proyecto Educativo Nacional al 2036. In Todos somos educadores (Issue 44). https:// www.cne.gob.pe/uploads/publicaciones/2020/ proyecto-educativo-nacional-al-2036.pdf

Declaración de Incheon. (2016). https://unescocat. org/wp-content/uploads/2019/04/ED2030_ DeclaracioIncheonCAT.pdf

Defensoría del Pueblo. (2020). El derecho a la educación inclusiva en el contexto de la emergencia sanitaria por el COVID-19. Informes Especiales $N^{\circ}$ 36-2020-DP. 24. https://www. turismo.gob.ec/wp-content/uploads/2020/05/ PROTOCOLO-ALOJAMIENTOS-V2.pdf

Diniz, D., Barbosa, L., y Dos Santos, W. (2009). Discapacidadm derechos humanos $y$ justicia. 64-77. https://www.scielo.br/j/ sur/a/fPMZfn9hbJYM7SzN9bwzysb/ ?format $=$ pdf\&lang $=$ es

Echeita, G., y Ainscow, M. (2011). La educación inclusiva como derecho. Pautas de Acción para el Desarrollo de una Revolución Pendiente. Tejuelo, 12, 26-46. http://www.uam.es/ personal_pdi/stmaria/sarrio/DOCUMENTOS,
ARTICULOS, PONENECIAS,/Educacion inclusiva como derecho. Ainscow y Echeita.pdf

Gallego-Ortega, J., y Rodríguez-Fuentes, A. (2021). Teaching Attitudes towards Students with Disabilities. Mathematics, 9(14), 1637. https:// doi.org/10.3390/math9141637

Gonzales-Sánchez, A., Trujillo, N., Pelayo, I., y Ocaña-Fernández, Y. (2021). Management in strengthening social- emotional competence during health emergencies: Gestão no fortalecimento da competência socioemocional durante emergências de saúde. Revista Tempos e Espaços Em Educação, 14(33), e15111. https:// doi.org/10.20952/revtee.v14i33.15111

Herrera, J. I., Parrilla, Á., Blanco, A., y Guevara, G. (2018). Teacher Training for Inclusive Education. A Challenge from the National University of Education in Ecuador. Revista Latinoamericana de Educación Inclusiva, 12(1), 21-38. https:// www.scielo.cl/pdf/rlei/v12n1/0718-7378rlei-12-01-00021.pdf

Jia, L., y Santi, M. (2021). Inclusive education for students with disabilities in the global COVID-19 outbreak emergency: some facts and thoughts from China. Disability and Society, 36(7), 1185-1190. https://doi.org/10.1080/0968 7599.2021 .1925226

Kamga, S. D. (2020). Covid-19 and the inclusion of learners with disabilities in basic education in South Africa: A critical analysis. African Human Rights Law Journal, 20(2), 558-583. https://doi. org/10.17159/1996-2096/2020/v20n2a9

Hernández Ríos, M. (2015). El Concepto de Discapacidad: De la Enfermedad al Enfoque de Derechos. Revista CES Derecho, 6(2), 46-59.

Nunes, S. da S., Saia, A. L., y Tavares, R. E. (2015). Educação Inclusiva: Entre a História, os Preconceitos, a Escola e a Família. Psicologia: Ciência e Profissão, 35(4), 1106- 1119. https:// doi.org/10.1590/1982-3703001312014

Llancavil Llancavil, D. (2016). Importancia de la Educación Inclusiva para el trabajo con niños con talento académico. Perspectiva Educacional, 55(1). https://doi.org/10.4151/07189729-vol.55iss.1-art.391 
Minedu. (2016). Currículo Nacional de la Educación Básica. http://www.minedu.gob.pe/curriculo/ pdf/curriculo-nacional-de-la-educacion-basica. pdf

Minedu. (2020). $N^{\circ} 093 R V$ Aprobar el documento normativo denominado "Orientaciones pedagógicas para el servicio educativo de Educación Básica durante el año 2020 en el marco de la emergencia sanitaria por el Coronavirus COVID-19". https:/www.gob.pe/institucion/ minedu/normas-legales/535987-093-2020minedu

Moreno Rodríguez, R., Tejada, A., y Díaz-Vega, M. (2020). COVID-19. Educación inclusiva $\mathrm{y}$ personas con discapacidad: fortalezas $\mathrm{y}$ debilidades de la teleeducación ColecciónNo Title No Title. Journal of Chemical Information and Modeling, 53(9), 1689-1699. http://hdl. handle.net/11181/6127

Murillo, F., y Duk, C. (2020). El Covid-19 y las Brechas Educativas. Revista Latinoamericana de Educación Inclusiva, 14(1), 11-13. https://doi. org/10.4067/s0718-73782020000100011

Nunes, S., Saia, A. y Tavares, R. (2015). Educação Inclusiva: Entre a História, os Preconceitos, a Escola e a Família. Psicologia: Ciência e Profissão, 35(4), 1106- 1119. https://doi. org/10.1590/1982-3703001312014

Peruano, D. El. (2020). En El Sector Público Ante La Emergencia Sanitaria Ocasionada Por El Covid-19 Decreto Legislativo No 1505. 4-6
Pozas, M., Letzel, V., y Schneider, C. (2021). 'Homeschooling in times of corona': exploring Mexican and German primary school students' and parents' chances and challenges during homeschooling. European Journal of Special Needs Education, 36(1), 35-50. https://doi.org/ 10.1080/08856257.2021.1874152

Rojo-Ramos, J., Manzano-Redondo, F., BarriosFernandez, S., Garcia-Gordillo, M. A., y Adsuar, J. C. (2021). A descriptive study of specialist and non-specialist teachers' preparation towards educational inclusion. International Journal of Environmental Research and Public Health, 18(14). https://doi.org/10.3390/ijerph18147428

Tabatadze, S., y Chachkhiani, K. (2021). COVID-19 and Emergency Remote Teaching in the Country of Georgia: Catalyst for Educational Change and Reforms in Georgia? Educational Studies - AESA, 57(1), 78-95. https://doi.org/1 $0.1080 / 00131946.2020 .1863806$

Valencia, A. (2018). Breve historia de las personas con discapacidad: De la Opresión a la Lucha por sus Derechos. Arctic Engineering, (Houston, U.S.A., II, New Yo, 39-46. https://www.scielo. $\mathrm{br} / \mathrm{j} / \mathrm{sur} / \mathrm{a} / \mathrm{fPMZfn} 9 \mathrm{hbJYM7SzN9bwzysb/}$ ?format=pdf\&lang=es 